Adam N. Letchford, Jens Lysgaard \& Richard W. Eglese

A Branch-and-Cut Algorithm for the Capacitated Open Vehicle Routing Problem

Logistics/SCM

Research Group 


\title{
A Branch-and-Cut Algorithm for the Capacitated Open Vehicle Routing Problem
}

\author{
Adam N. Letchford*, Jens Lysgaard ${ }^{+}$and Richard W. Eglese* \\ ${ }^{*}$ Department of Management Science, Lancaster University, \\ Lancaster LA1 4YW, England \\ e-mail: \{A.N.Letchford,R.Eglese\}@lancaster.ac.uk \\ ${ }^{+}$Logistics/SCM Research Group, Department of Business Studies, \\ Aarhus School of Business, Fuglesangs Allé 4, DK-8210 Aarhus V, Denmark \\ e-mail: lys@asb.dk
}

March 2006

\begin{abstract}
In open vehicle routing problems, the vehicles are not required to return to the depot after completing service. In this paper, we present the first exact optimization algorithm for the open version of the well-known capacitated vehicle routing problem (CVRP). The algorithm is based on branch-and-cut. We show that, even though the open CVRP initially looks like a minor variation of the standard CVRP, the integer programming formulation and cutting planes need to be modified in subtle ways.

Computational results are given for several standard test instances, which enables us for the first time to assess the quality of existing heuristic methods, and to compare the relative difficulty of open and closed versions of the same problem.
\end{abstract}

Key words: vehicle routing, branch-and-cut, separation.

\section{Introduction}

In the classical version of Vehicle Routing Problems (VRPs), the vehicles are required to return to the depot after completing service (see for example Toth \& Vigo, 2002). In open VRPs, however, the vehicles need not do so. As a result, the vehicle routes are not closed paths but open ones, starting at the depot and ending at one of the customers. Figure 1, which shows the optimal solutions to both the open and closed version for the same input data (all customers have unit demands and the vehicle capacity is five units), illustrates the fact that, in general, the optimal solution for the open version of a VRP 

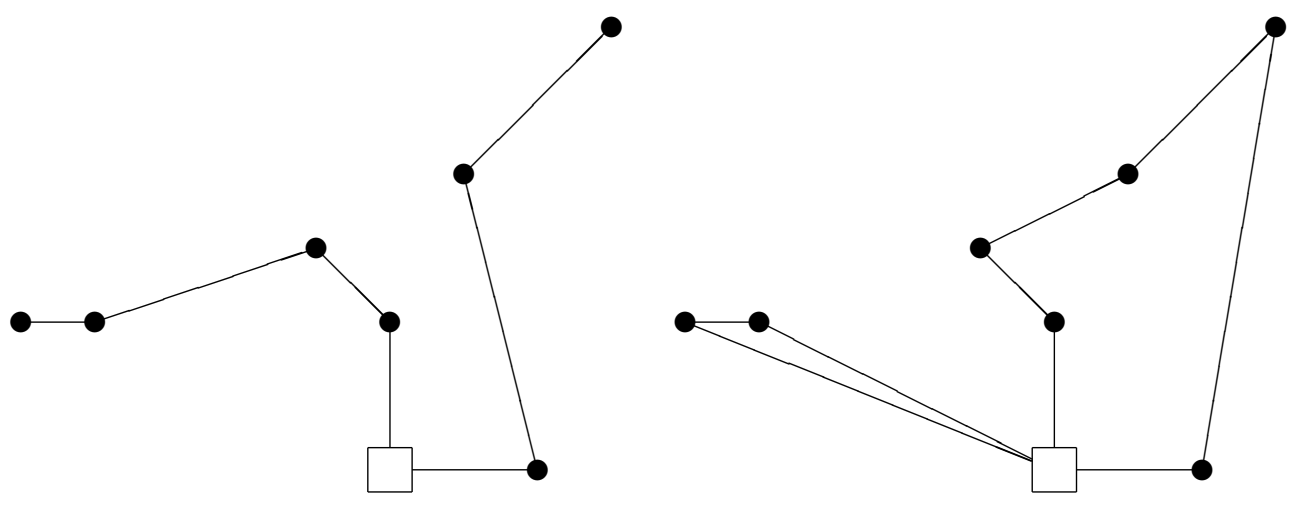

Figure 1: Open versus closed routes with different clusterings of customers.

can be quite different from that for the closed version. (Throughout this paper, the depot is represented by a white square and the customers by black circles.)

At first sight, having open routes instead of closed ones looks like a minor modification. Indeed, if travel costs are asymmetric, there is essentially no difference between the open and closed versions: to transform the open version into the closed one, it suffices to set the cost to zero for traveling from any customer to the depot. However, if travel costs are symmetric, things are more subtle. Indeed, we prove in the next section that, somewhat surprisingly, the open version turns out to be more general than the closed one, in the sense that any closed VRP on $n$ customers can be transformed into an open VRP on $n$ customers, but there is no transformation in the reverse direction.

Moreover, there are many practical applications in which open VRPs naturally arise. This happens for example when a company does not own a vehicle fleet and all its deliveries from a central depot are undertaken by hired vehicles that are not obliged to return to the depot. In such situations, the cost of the distribution may be proportional to the distance travelled while loaded. A practical case study of this type is described in Tarantilis et al. (2005). The same model can also be used for pick-ups, where vehicles start empty at any customer and must pick up the demands of each customer on their route and deliver them to the depot.

There are also applications where the vehicles start at the depot, deliver to a set of customers and then are required to visit the customers in reverse order, picking up items that are required to be backhauled to the depot. If, for each customer, the pick-up 
demand is no larger than the delivery demand, then an open VRP model can be used. An application of this type for an air express courier is mentioned by Schrage (1981) in an early article describing features of practical routing problems.

Two further areas of application are described by Fu et al. (2005). The first involves the planning of train services, starting or ending at the Channel Tunnel. The second involves planning a set of school bus routes where in the morning pupils are picked up at various locations and brought to school, and in the afternoon, the routes are reversed to take pupils home. Bodin et al. (1983) includes a description of a problem of express airmail distribution in the USA, that is essentially an open pick-up and delivery VRP with capacity constraints and time windows.

Open VRPs are easily seen to be strongly $\mathcal{N} \mathcal{P}$-hard by reduction from the Hamiltonian path problem. Research on open VRPs has therefore up to now concentrated on devising effective heuristics for solving them. For the version involving only capacity constraints, Sariklis and Powell (2000) presented a two-phase heuristic involving minimum spanning trees, and Tarantilis et al. (2005) present a metaheuristic of the threshold-accepting type. For a more general variant involving both capacity and route-length constraints, Brandão (2004) and Fu et al. (2005) describe tabu search heuristics, Li et al. (2006) present a recordto-record travel heuristic, and Pisinger \& Ropke (2006) present an adaptive neighborhood search heuristic.

In this paper we present the first exact optimization algorithm for the Capacitated Open Vehicle Routing Problem (COVRP), which is defined as follows. A complete undirected graph $G=(V, E)$ is given, with $V=\{0, \ldots, n\}$. Vertex 0 represents the depot, the other vertices represent customers. The cost of travel from vertex $i$ to vertex $j$ is denoted by $c_{i j}$, and we assume costs are symmetric, so $c_{i j}=c_{j i}$. A fleet of $K$ identical vehicles, each of capacity $Q>0$, is given. Each customer $i$ has a demand $q_{i}$, with $0<q_{i} \leq Q$. Each customer must be serviced by a single vehicle and no vehicle may serve a set of customers whose total demand exceeds its capacity. Each vehicle route must start at the depot and end at the last customer it serves. The objective is to define the set of vehicle routes that minimizes the total costs.

Other variants of the COVRP can be defined, in which the vehicle fleet size is free, and possibly a fixed cost is associated with the use of a vehicle. Such variants can easily be handled with minor modifications of the methods that we propose.

The structure of the remainder of the paper is as follows. In the next section, we give an integer programming formulation of the COVRP and present some valid inequalities. 
It will be seen that more complex inequalities are needed for the open version than for the closed version. Then, in the following section, we describe the ingredients of our branchand-cut algorithm. Extensive computational results are given in the following section, which enable us for the first time to assess the quality of existing heuristic methods, and to compare the relative difficulty of open and closed versions of the same problem. Some concluding remarks are given in the final section.

\section{Formulation and Valid Inequalities}

\section{Formulation}

The COVRP is clearly a special case of the asymmetric CVRP (ACVRP), in which, for any $i, j \in V, c_{i j}$ is permitted to be different from $c_{j i}$. Hence, it would be possible to use any integer programming formulation of the ACVRP (for example, that of Fischetti et al., 1994) to solve the COVRP. However, this would mean that effectively we were treating each (undirected) edge as two (directed) arcs, which would lead to a formulation of the COVRP with twice as many variables as our formulation of the CVRP. This seems unnecessary, given that, in the COVRP, $c_{i j}=c_{j i}$ when $i$ and $j$ are customers.

A more parsimonious formulation of the COVRP can be obtained by modifying the standard formulation of the CVRP. To explain this clearly, it is helpful to recall the following details of the CVRP formulation.

Let $V_{c}=V \backslash\{0\}$ denote the set of customers. Given a set $S \subseteq V_{c}$, let $q(S)$ denote $\sum_{i \in S} q_{i}, \delta(S)$ denote the set of edges in $G$ with exactly one end-vertex in $S, E(S)$ denote the set of edges in $G$ with both end-vertices in $S$, and $k(S)$ denote $\lceil q(S) / Q\rceil$. Obviously, $k(S)$ is a lower bound on the minimum number of vehicles required to serve the customers in $S$. Let $x_{i j}$ represent the number of times a vehicle travels between vertices $i$ and $j$. (Because the problem is undirected, $x_{i j}$ and $x_{j i}$ represent the same variable.) Finally, given an arbitrary $F \subseteq E, x(F)$ will denote $\sum_{e \in F} x_{e}$. Then the standard (so-called two-index) formulation of the CVRP is (Laporte, Nobert \& Desrochers, 1985):

$$
\text { Minimize } \quad \sum_{e \in E} c_{e} x_{e}
$$

Subject to:

$$
\begin{array}{cl}
x(\delta(\{i\}))=2 & (i=1, \ldots, n) \\
x(\delta(S)) \geq 2 k(S) & \left(S \subseteq V_{c},|S| \geq 2\right) \\
x(\delta(\{0\}))=2 K &
\end{array}
$$




$$
\begin{array}{cl}
x_{i j} \in\{0,1\} & (1 \leq i<j \leq n) \\
x_{0 i} \in\{0,1,2\} & (i=1, \ldots, n) .
\end{array}
$$

The degree equations (1) ensure that customers are visited exactly once. The rounded capacity inequalities (2) impose the vehicle capacity restrictions and also ensure that the routes are connected. They can be re-written, using the degree equations, in the alternative form

$$
x(E(S)) \leq|S|-k(S) .
$$

The equation (3) ensures that exactly $K$ vehicles are used. Finally, constraints (4) and (5) are the integrality conditions. Note that the variables $x_{0 i}$ are permitted to take the value 2 , to allow routes in which a vehicle serves a single customer.

To adapt this formulation to the COVRP, we simply treat each edge incident on the depot as a pair of directed arcs, as follows. For each $i \in V_{c}$, instead of defining the undirected variable $x_{0 i}$, we define the binary variable $y_{0 i}$, which takes the value 1 if and only if a vehicle travels directly from the depot to $i$, and the variable $y_{i 0}$, which takes the value 1 if and only if a vehicle travels directly from $i$ to the depot. We also use the notation $y^{-}(S)=\sum_{i \in S} y_{0 i}$ and $y^{+}(S)=\sum_{i \in S} y_{i 0}$. Finally, for any $S \subset V_{c}$ we use the notation $\bar{S}=V_{c} \backslash S$ and $\bar{\delta}(S)=\{\{i, j\}: i \in S, j \in \bar{S}\}$.

If we adapt the above formulation to the COVRP in a straightforward manner, we obtain the following integer program:

$$
\text { Minimize } \quad \sum_{e \in E\left(V_{c}\right)} c_{e} x_{e}+\sum_{i \in V_{c}} c_{0 i} y_{0 i}
$$

Subject to:

$$
\begin{array}{cl}
x(\bar{\delta}(i))+y_{0 i}+y_{i 0}=2 & (i=1, \ldots, n) \\
x(\bar{\delta}(S))+y^{-}(S)+y^{+}(S) \geq 2 k(S) & \left(S \subseteq V_{c},|S| \geq 2\right) \\
y^{-}\left(V_{c}\right)=K & \\
y^{+}\left(V_{c}\right)=K & \\
x_{i j} \in\{0,1\} & (1 \leq i<j \leq n) \\
y_{0 i}, y_{i 0} \in\{0,1\} & (i=1, \ldots, n) .
\end{array}
$$

The constraints (7), (8) are straighforward adaptations of the degree equations and rounded capacity inequalities, respectively. The inequalities (8) can again be re-written in the form $x(E(S)) \leq|S|-k(S)$. The constraints (9) and (10) ensure that exactly $K$ vehicles leave and enter the depot. Finally, constraints (11) and (12) ensure that all variables are binary. (There is no longer any need to allow any variables to take the value 2.) 


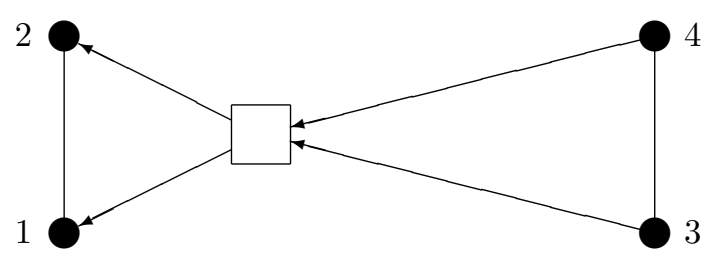

Figure 2: Invalid integer solution.

Perhaps surprisingly, the above integer program does not represent a valid formulation for the COVRP. Figure 2 shows a solution to the above integer program for a small COVRP instance with $n=4$, which does not represent a valid solution to the COVRP.

To prevent invalid solutions of this kind, it is necessary to add the following constraints to the formulation:

$$
x(\bar{\delta}(S))+y^{+}(S) \geq y^{-}(S) \quad\left(S \subseteq V_{c},|S| \geq 2\right) .
$$

We call these constraints balancing inequalities. The fact that they are valid, and sufficient to ensure feasibility, follows from the conditions of Ford and Fulkerson (1962) for a mixed graph to be Eulerian. (Some similar inequalities were introduced by Nobert and Picard, 1996, in the context of the so-called Mixed Chinese Postman Problem.)

The invalid solution above, for example, violates the balancing inequality with $S=$ $\{1,2\}$, which takes the form

$$
x_{13}+x_{14}+x_{23}+x_{24}+y_{10}+y_{20} \geq y_{01}+y_{02} .
$$

It turns out that, once the balancing inequalities have been added to the formulation, the equation (9) is redundant.

Note that there are an exponential number of balancing inequalities. The need for balancing inequalities, which have no counterpart for the standard CVRP, suggests that the COVRP is a more complex problem than the CVRP. This is confirmed by the following definition and proposition.

Definition 1 The partially asymmetric CVRP (PACVRP) is the generalization of the $C V R P$ in which the cost of travel $c_{0 i}$ is permitted to be different from $c_{i 0}$. 
Obviously, the PACVRP is intermediate in generality between the CVRP and ACVRP. The following result is less obvious.

Proposition 1 The COVRP and the PACVRP are equivalent.

Proof: Any COVRP instance is clearly a PACVRP instance. Now, suppose we are given a PACVRP instance on $n$ vertices, with symmetric travel costs $c_{i j}$ for all $\{i, j\} \in E\left(V_{c}\right)$ and asymmetric travel costs $c_{0 i}, c_{i 0}$ for all $i \in V_{c}$. Now let $M$ be an arbitrary constant. Due to the presence of the degree equations (7), (9) and (10), the optimal solution to the PACVRP is unchanged if we replace the original travel costs $c_{i j}$ with modifed $\operatorname{costs} c_{i j}^{\prime}$, defined as follows:

- for all $\{i, j\} \in E\left(V_{c}\right), c_{i j}^{\prime}=c_{i j}-c_{i 0}-c_{j 0}+M$,

- for all customers $i, c_{0 i}^{\prime}=c_{0 i}$ and $c_{i 0}^{\prime}=0$.

If we choose $M$ appropriately, the transformed $\operatorname{costs} c_{i j}^{\prime}$ will be non-negative. (An appropriate value of $M$ is $\max _{1 \leq i<j \leq n}\left\{c_{i 0}+c_{j 0}-c_{i j}\right\}$.) Since the costs $c_{i 0}^{\prime}$ are now zero, we have a COVRP instance.

The algorithm we propose in this paper can therefore be used to solve instances of the PACVRP

We remark that an alternative integer programming formulation for the COVRP can be obtained by eliminating the variables $y_{i 0}$, which do not appear in the objective function, via the equations (7). Although the resulting formulation has $n$ fewer variables, it is harder to understand and analyse and, more importantly, has a higher density (proportion of nonzeroes), which is unattractive from a computational point of view. For these reasons, we prefer to use the original formulation.

In the following subsections, we examine various valid inequalities for the integer polyhedron associated with the above formulation.

\section{Symmetric inequalities}

From a polyhedral point of view, the COVRP is similar to the CVRP. The following proposition, which is trivial to prove, shows that any valid inequality for the CVRP yields a valid inequality for the COVRP.

Proposition 2 Let $\sum_{0 \leq i<j \leq n} \alpha_{i j} x_{i j} \leq \beta$ be valid for the CVRP. Then $\sum_{1 \leq i<j \leq n} \alpha_{i j} x_{i j}+$ $\sum_{i=1}^{n} \alpha_{0 i}\left(y_{0 i}+y_{i 0}\right) \leq \beta$ is valid for the COVRP. 
We call inequalities obtained in this way symmetric. A simple example of a class of symmetric inequalities is given by the inequalities (8), which are clearly the symmetric version of the rounded capacity inequalities (2). Other valid inequalities for the CVRP include, for example, the homogeneous multistar and partial multistar, generalized large multistar, framed capacity, strengthened comb, and hypotour inequalities. See Augerat (1995), Letchford et al. (2002), Lysgaard et al. (2004) and Naddef \& Rinaldi (2002) for details. From Proposition 2, these all have valid counterparts for the COVRP.

\section{Asymmetric inequalities}

We say that a valid inequality $\alpha x+\beta y \geq \gamma$ for the COVRP is asymmetric if there exists at least one $i \in V_{c}$ such that $\beta_{0 i} \neq \beta_{i 0}$. The existence and necessity of the balancing inequalities shows that there exist non-redundant asymmetric inequalities. This should be expected, since the COVRP is a generalization of the CVRP.

Using the degree equations, it is possible to write the balancing inequality in a variety of forms. In particular, the balancing inequality for $S$ is equivalent to $x(\bar{\delta}(\bar{S}))+y^{-}(\bar{S}) \geq$ $y^{+}(\bar{S})$. Therefore, there is no need for a 'reversed' form of the balancing inequality, of the form $x(\bar{\delta}(S))+y^{-}(S) \geq y^{+}(S)$, since this is equivalent to the balancing inequality on $\bar{S}$.

Unfortunately, the addition of all symmetric inequalities to the bounds, degree equations and balancing inequalities still does not give a complete description of the COVRP polyhedron. Suppose $n=6, Q=5$ and $q_{i}=1$ for $i=1, \ldots, 6$, and consider the fractional point displayed in Figure 3. (The dotted lines represent edges whose variables have value $1 / 2$.) It is easy to check that it satisfies the bounds, degree equations and balancing inequalities. Moreover, it satisfies all symmetric inequalities. To see this, note that, if we replace the directed arcs with undirected edges, the resulting fractional point for the CVRP is a convex combination of the two integral points displayed in Figure 4.

The fractional point displayed in Figure 3 can be cut off by the inequality $x_{12}+x_{15}+$ $x_{25}+x_{56}+y_{01}+y_{20} \leq 4$. This inequality, which is easily seen to be valid for the COVRP, is a special case of a class of inequalities which we call mixed strengthened comb inequalities. These inequalities are presented in the following theorem. 


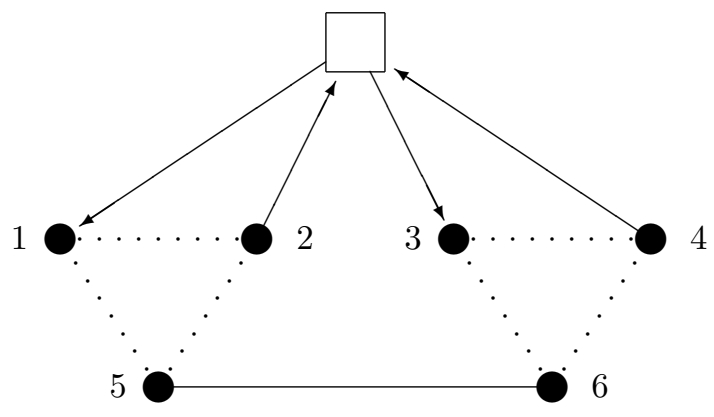

Figure 3: Fractional point satisfying all balancing and symmetric inequalities.

Theorem 1 Let $H \subset V_{c}$ (the handle) and $T_{1}, \ldots, T_{t} \subset V$ (the teeth) be such that:

- every tooth properly intersects with the handle, i.e., $T_{i} \cap H$ and $T_{i} \backslash H$ are non-empty for all $i$;

- if any pair of teeth intersect, then either all vertices in the intersection lie in the handle or all lie outside, i.e., for $1 \leq i<j \leq t$, either $T_{i} \cap T_{j} \subset H$ or $T_{i} \cap T_{j} \cap H=\emptyset$.

Moreover, let the index set $\{1, \ldots, t\}$ be partitioned into sets $\mathcal{N}, \mathcal{D}, \mathcal{S}, \mathcal{R}$ such that $0 \notin T_{i}$ for all $i \in \mathcal{N}$ and $0 \in T_{i}$ for all $i \in \mathcal{D} \cup \mathcal{S} \cup \mathcal{R}$. We call $T_{i}$ a normal tooth if $i \in \mathcal{N} \cup \mathcal{D}$, a sending tooth if $i \in \mathcal{S}$ and a receiving tooth if $i \in \mathcal{R}$. Now, for any tooth $T_{i}$, we define:

$$
\gamma\left(T_{i}\right)= \begin{cases}k\left(T_{i}\right)+k\left(T_{i} \cap H\right)+k\left(T_{i} \backslash H\right) & \text { if } i \in \mathcal{N}, \\ k\left(V \backslash T_{i}\right)+k\left(T_{i} \cap H\right)+k\left(V \backslash\left(T_{i} \backslash H\right)\right) & \text { if } i \in \mathcal{D}, \\ k\left(T_{i} \cap H\right)+2 & \text { if } i \in \mathcal{S} \cup \mathcal{R} .\end{cases}
$$

Then, if $\sum_{i=1}^{t} \gamma\left(T_{i}\right)$ is odd, the mixed comb inequality

$$
\begin{gathered}
x(E(H))+\sum_{i \in \mathcal{N}} x\left(E\left(T_{i}\right)\right)+\sum_{i \in \mathcal{D} \cup \mathcal{S} \cup \mathcal{R}} x\left(E\left(T_{i} \backslash\{0\}\right)\right)+\sum_{i \in \mathcal{D} \cup \mathcal{S}} y^{+}\left(T_{i} \backslash\{0\}\right) \\
+\sum_{i \in \mathcal{D} \cup \mathcal{R}} y^{-}\left(T_{i} \backslash\{0\}\right) \leq|H|+\sum_{i=1}^{t}\left|T_{i}\right|+|\mathcal{D}|(K-1)-\left\lceil\sum_{i=1}^{t} \gamma\left(T_{i}\right) / 2\right\rceil
\end{gathered}
$$

is valid for the COVRP.

The proof of this theorem is in the Appendix.

The mixed strengthened comb inequalities reduce to ordinary strengthened comb inequalities (Lysgaard et al., 2004) when there are no sending and receiving teeth. The fractional point displayed in Figure 3 violates the mixed comb inequality with handle $H=\{1,2,5\}$, normal tooth $\{5,6\}$, receiving tooth $\{0,1\}$ and sending tooth $\{0,2\}$. 

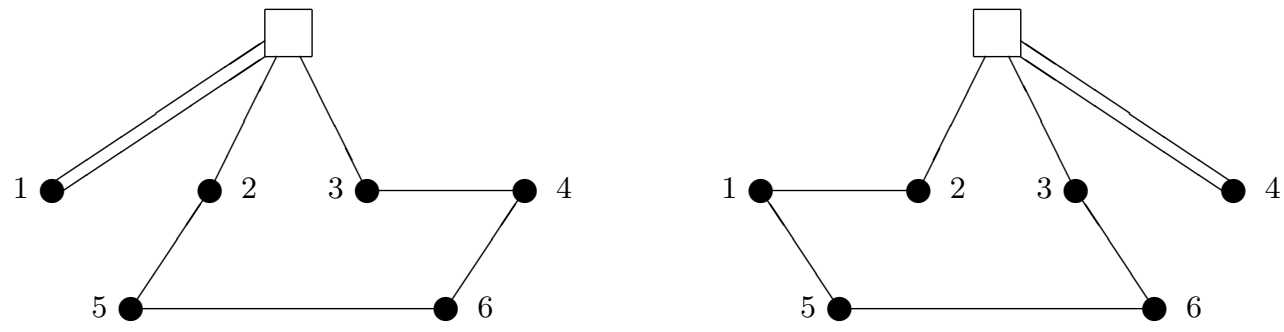

Figure 4: Two feasible CVRP solutions.

For many instances of the CVRP or COVRP, the number of vehicles $K$ is fixed at the minimum possible, which often equals $k\left(V_{c}\right)$. In such a case, a lower bound on the amount which must be loaded onto any vehicle in any feasible solution is $q_{\min }=q\left(V_{c}\right)-Q(K-1) \geq$ 0 . It is easy to show that it is never worthwhile having a vertex set as a sending or receiving tooth unless the total demand of that set is at least $q_{m i n}$, and that it is not worthwhile having a customer set $T$ as a normal tooth if $k(T)=k(T \cap H)+k(T \backslash H)$.

\section{The Branch-and-Cut Algorithm}

Our implementation of the branch-and-cut algorithm for the COVRP follows to a large extent the implementation of our branch-and-cut algorithm for the CVRP, which was described in detail in Lysgaard et al. (2004). In particular, the ingredients for branching, node selection, cut pool management, and basis reconstruction are implemented as in Lysgaard et al. (2004).

As such, we focus here on those parts of our implementation for the COVRP that have no direct counterpart in our implementation for the CVRP. In the next three subsections we describe our separation algorithms for symmetric, balancing and mixed strengthened comb inequalities, respectively, and after that we describe the overall separation strategy.

We let $\left(x^{*}, y^{*}\right)$ denote the current LP solution satisfying (7), (9), (10), and the bounds implied by (11), (12). 


\section{Separation of symmetric inequalities}

As noted in Proposition 2, valid inequalities for the COVRP can be obtained by a simple reformulation of valid inequalities for the CVRP. This allows us to exploit the fact that effective separation algorithms have been developed for several classes of inequalities for the CVRP. In particular, in Letchford et al. (2002) and Lysgaard et al. (2004), we described effective separation algorithms for rounded capacity, homogeneous multistar and partial multistar, generalized large multistar, framed capacity, strengthened comb and hypotour inequalities. These algorithms have been made publicly available (Lysgaard, 2003). We use these separation routines in our branch-and-cut algorithm, but with a modification to the strengthened comb separation routine to cope with the existence of sending and receiving teeth.

For details of these routines and the issue of sparse representations of the inequalities we refer to Letchford et al. (2002), Lysgaard (2003) and Lysgaard et al. (2004).

\section{Separation of balancing inequalities}

The balancing inequalities can easily be separated in polynomial time by reduction to a max-flow/min-cut problem. We add $y^{-}(\bar{S})$ to both sides of (13) to obtain:

$$
x(\bar{\delta}(S))+y^{+}(S)+y^{-}(\bar{S}) \geq K .
$$

Thus, a balancing inequality is violated for a set $S$ if and only if the left hand side of (15), computed with respect to $\left(x^{*}, y^{*}\right)$, is less than $K$. So, construct a directed graph $\hat{G}=(\hat{V}, \hat{A})$ in the following way. The vertex set $\hat{V}$ is set to $V \cup\{n+1\}$. For each $\{i, j\} \in E\left(V_{c}\right)$ such that $x_{i j}^{*}>0$, insert two $\operatorname{arcs}(i, j)$ and $(j, i)$ into $\hat{A}$ and give them a capacity of $x_{i j}^{*}$. For each $i \in V_{c}$, such that $y_{0 i}^{*}>0$ (respectively, $y_{i 0}^{*}>0$ ), insert the arc $(0, i)$ (respectively, $(i, n+1))$ into $\hat{A}$ and give it a capacity of $y_{0 i}^{*}$ (respectively, $y_{i 0}^{*}$ ). Then, send a maximum flow in $\hat{G}$ from 0 to $n+1$, to find a minimum capacity $(0, n+1)$-cut. If the cut has a capacity less than $K$, a balancing inequality is violated, and the desired set $S$ is given by the customer set on the same shore of the cut as 0 .

It often happens that the subgraph of the support graph induced by the edges in $E(S)$ is disconnected. This defines a partition of $S$ into sets, say $S_{1}, \ldots, S_{t}$. In this case, it is easy to show that the sum of the violations of the balancing inequalities for the sets $S_{1}, \ldots, S_{t}$ is equal to the violation of the original balancing inequality. Figure 5 illustrates this possibility. The separation algorithm returns the set $S=\{1,2,3,4\}$, for 


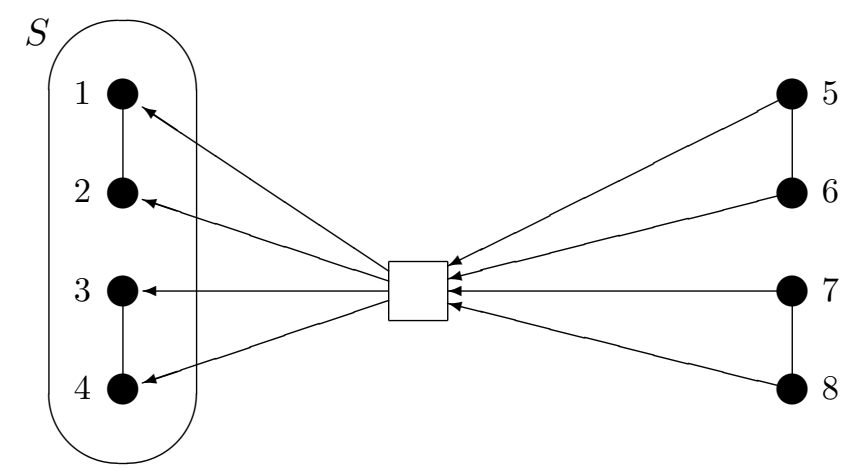

Figure 5: Decomposing a balancing inequality.

which the balancing inequality is violated by 4 . There are two components, $S_{1}=\{1,2\}$ and $S_{2}=\{3,4\}$, for each of which the balancing inequality is violated by 2 .

Thus, if the exact separation algorithm returns a violated balancing inequality we can often find a number of other violated balancing inequalities with negligible additional work. (A similar observation was made by Nobert and Picard, 1996.)

The above separation algorithm, using for example the pre-flow push max-flow algorithm of Goldberg and Tarjan (1988), runs in $\mathcal{O}\left(n m \log \left(n^{2} / m\right)\right)$ time, where $m$ is the number of variables which are positive at $\left(x^{*}, y^{*}\right)$. Faster heuristics for separation can be devised, based for example on connected components, but this proved to be unnecessary in our computational experiments.

\section{Separation of mixed strengthened comb inequalities}

As mentioned above, we described in Lysgaard et al. (2004) an effective heuristic for the separation of strengthened comb inequalities. This heuristic relied in part on the fact that the separation problem becomes polynomially solvable in the special case in which each tooth has cardinality 2. In this case, the strengthened comb inequalities reduce to a variant of the 2-matching inequalities of Edmonds (1965), and the separation problem can therefore be solved with a minor modification of the algorithm of Padberg and Rao (1982). The separation heuristic for the case of general strengthened comb inequalities 
can be summarized as follows:

- Apply shrinking to the support graph, in such a way that a violated strengthened comb inequality in the shrunk support graph corresponds to a violated strengthened comb inequality in the original.

- Run a heuristic, based on connected components, to generate a set of 'seed' strengthened comb inequalities, not necessarily violated, such that each tooth has cardinality two.

- For each such 'seed' strengthened comb inequality, enlarge the teeth in a greedy way in an attempt to increase the violation (or, equivalently, decrease the slack).

- If no violated inequality has been found, run the modified Padberg-Rao algorithm to find more 'seed' strengthened comb inequalities, and repeat the above greedy procedure.

- If any of the resulting strengthened comb inequalities are violated, output the corresponding violated strengthened comb inequalities for the original graph.

To enable this heuristic to separate the more general mixed strengthened comb inequalities, it was necessary to further modify the Padberg-Rao algorithm, to cope with sending and receiving teeth of cardinality 2 . To do this, we note that, for any $i \in V_{c}$, we have three cases:

- Case 1: $q_{i}<q_{\min }$. In this case, it could be worthwhile making $\{0, i\}$ a tooth in $\mathcal{D}$, and, if we did, it would contribute $1-\left(y_{0 i}^{*}+y_{i 0}^{*}\right)$ to the slack of the inequality.

- Case 2: $q_{i} \geq q_{\min }$ and $y_{i 0}^{*} \geq y_{0 i}^{*}$. In this case, it could be worthwhile making $\{0, i\}$ a tooth in $\mathcal{S}$, which would contribute $1-y_{i 0}^{*}$ to the slack.

- Case 3: $q_{i} \geq q_{\min }$ and $y_{i 0}^{*}<y_{0 i}^{*}$. In this case, it could be worthwhile making $\{0, i\}$ a tooth in $\mathcal{R}$, which would contribute $1-y_{0 i}^{*}$ to the slack.

On the other hand, if we did not use $\{0, i\}$ as a tooth, but $i$ belonged to the handle, this would contribute $y_{0 i}^{*}+y_{i 0}^{*}$ to the slack. Thus, we take these four possibilities into account when splitting edges in the Padberg-Rao procedure.

In addition, we modified the greedy procedure for enlarging teeth. If a 'seed' tooth contains the depot, we have three options: it can be put into $\mathcal{D}, \mathcal{S}$ or $\mathcal{R}$. We run the greedy enlargement process three times accordingly. The chosen category for the tooth is then the one resulting in the minimum slack (i.e., the maximum violation). 


\section{Separation strategy}

In our algorithm for the CVRP, we called our separation routines in the following order. First, we called rounded capacity separation. If this failed to find a rounded capacity violated by a significant amount, we called framed capacity separation. If this also failed, we proceeded to the separation of multistar, strengthened comb and hypotour inequalities. Finally, we also permitted a round of Gomory mixed-integer cuts to be generated.

For the COVRP, we use essentially the same separation strategy. The only differences are that i) we call both rounded capacity and balancing separation at the first stage, ii) we replace our strengthened comb separation heuristic with its mixed counterpart, and iii) to reduce implementational effort, we do not generate Gomory cuts.

\section{Computational Experiments}

Our algorithm has been coded in the $\mathrm{C}$ programming language using the Microsoft Visual $\mathrm{C}++$ v. 6.0 compiler. For solving LPs we have used the CPLEX callable library v. 9.0. All experiments have been done on a PC with a $1.6 \mathrm{GHz}$ Intel Pentium M processor and 512 MB of RAM running Microsoft Windows XP.

In this section we show the performance of the entire algorithm on both closed and open versions of several instances. Instead of using our algorithm from Lysgaard et al. (2004) when solving CVRPs, we use to an as large extent as possible the same code for solving both COVRPs and CVRPs, in order to eliminate the potential effects of differences in implementation details. Specifically, given our COVRP algorithm we obtain a CVRP algorithm by i) changing $c_{i 0}$ from 0 to $c_{0 i}$ for $i=1, \ldots, n$, ii) deactivating balancing inequalities, and iii) not permitting sending or receiving teeth in comb inequalities.

We have done our experiments on the so-called A, B, E, F, M, and P benchmark CVRP instances, which are available at www . branchandcut . org (accessed 1st February 2006). Most of the closed versions have already been solved to optimality, see for example Lysgaard et al. (2004) and Fukasawa et al. (2006). In all of these instances, the vertices are taken to be points located in the Euclidean plane. The cost of an edge is then taken to be equal to the Euclidean distance between its end-vertices. We had to make a decision concerning precision in the computation of these distances. As discussed in Toth \& Vigo (2002), some authors represent the distances as floating point numbers within their algorithms, and then report the cost of the solutions to a fixed precision, such as one decimal place. Other authors, however, round the distances to integers following the TSPLIB standard 


\begin{tabular}{|c|c|c|c|c|c|c|c|}
\hline \multirow[b]{3}{*}{ Name } & \multicolumn{5}{|c|}{ COVRP } & \multirow{2}{*}{\multicolumn{2}{|c|}{$\frac{\text { CVRP }}{\text { Branch \& cut }}$}} \\
\hline & \multicolumn{2}{|c|}{ Root node } & \multicolumn{3}{|c|}{ Branch \& cut } & & \\
\hline & LB & Time & LB & Tree size & Time & LB & Time \\
\hline A-n32-k5 & 487.306 & 0.1 & $487.306^{*}$ & 1 & 0.1 & $787.082^{*}$ & 3 \\
\hline A-n33-k5 & 424.543 & 0.2 & $424.543^{*}$ & 1 & 0.2 & $662.11^{*}$ & 2 \\
\hline A-n33-k6 & 455.794 & 0.4 & $462.433^{*}$ & 10 & 0.8 & $742.693^{*}$ & 1 \\
\hline A-n34-k5 & 494.313 & 0.3 & $508.173^{*}$ & 56 & 3 & $780.936^{*}$ & 2 \\
\hline A-n36-k5 & 510.011 & 1 & $519.455^{*}$ & 36 & 3 & $802.132^{*}$ & 4 \\
\hline A-n $37-\mathrm{k} 5$ & 482.858 & 0.5 & $486.243^{*}$ & 14 & 0.9 & $672.465^{*}$ & 3 \\
\hline A-n37-k6 & 576.64 & 1 & $581.073^{*}$ & 14 & 2 & $950.852^{*}$ & 52 \\
\hline A-n38-k5 & 487.597 & 0.4 & $497.997^{*}$ & 48 & 3 & $733.946^{*}$ & 5 \\
\hline A-n39-k5 & 541.523 & 1 & $549.684^{*}$ & 86 & 9 & $828.989^{*}$ & 36 \\
\hline A-n39-k6 & 526.528 & 0.3 & $533.066^{*}$ & 17 & 2 & $833.205^{*}$ & 6 \\
\hline $\mathrm{A}-\mathrm{n} 44-\mathrm{k} 7$ & 602.733 & 1 & $617.385^{*}$ & 256 & 29 & $938.181^{*}$ & 45 \\
\hline A-n $45-\mathrm{k} 6$ & 603.162 & 2 & $648.669 *$ & 5101 & 1121 & $944.876^{*}$ & 13 \\
\hline A-n $45-\mathrm{k} 7$ & 655.541 & 3 & $685.156^{*}$ & 5742 & 1846 & $1146.772^{*}$ & 1131 \\
\hline A-n46-k7 & 578.962 & 0.4 & $583.538^{*}$ & 6 & 0.8 & $917.724^{*}$ & 12 \\
\hline A-n48-k7 & 651.143 & 1 & $669.826^{*}$ & 175 & 34 & $1074.338^{*}$ & 46 \\
\hline A-n53-k7 & 643.795 & 2 & $655.184^{*}$ & 70 & 18 & $1012.249 *$ & 90 \\
\hline A-n54-k7 & 681.666 & 4 & $709.272^{*}$ & 1966 & 762 & $1171.682^{*}$ & 2007 \\
\hline A-n 55-k9 & 646.642 & 2 & $669.06^{*}$ & 1978 & 472 & $1074.464^{*}$ & 43 \\
\hline A-n60-k9 & 756.362 & 6 & 780.409 & 8048 & 3600 & 1341.506 & 3600 \\
\hline A-n61-k9 & 639.362 & 4 & 664.538 & 7440 & 3600 & 1038.135 & 3600 \\
\hline A-n62-k8 & 757.755 & 4 & $783.176^{*}$ & 2523 & 1231 & 1286.145 & 3600 \\
\hline A-n63-k9 & 911.835 & 10 & 935.748 & 5466 & 3600 & 1610.838 & 3600 \\
\hline A-n63-k10 & 745.936 & 5 & 773.995 & 7692 & 3600 & 1303.6 & 3600 \\
\hline A-n64-k9 & 811.983 & 4 & 837.092 & 6772 & 3600 & 1380.109 & 3600 \\
\hline A-n65-k9 & 711.219 & 3 & $728.591^{*}$ & 170 & 58 & $1181.687^{*}$ & 284 \\
\hline A-n69-k9 & 732.273 & 5 & $757.764^{*}$ & 4644 & 2659 & 1152.411 & 3600 \\
\hline A-n80-k10 & 1019.84 & 9 & 1038.504 & 4372 & 3600 & 1736.875 & 3600 \\
\hline
\end{tabular}

Table 1: Results for the A instances.

of Reinelt (1991). Our algorithm can cope with both versions. Since papers on the OVRP tend to report results only for the floating point versions, in this paper we do the same.

Tables 1 to 4 report the results. In each table, the first column gives the instance name. The name indicates the source of the instance, the number of vertices, and the number of vehicles, which for all of these instances is fixed at the minimum possible. For example, E-n22-k4 is taken from Christofides \& Eilon (1969), and has 22 vertices and 4 vehicles. The next two columns give, for the open version, the lower bound at the root node of the branch-and-bound tree and the time taken to obtain it. All times in the tables are given in seconds. The next three columns give, again for the open version, the best lower bound found within 1 hour, the number of branches processed within 1 hour, and the time taken to solve the instance to optimality if the instance was solved in less than an hour. An asterisk indicates that the instance has been solved to optimality. The last two columns give the lower bound and time for the closed version of the instance. 


\begin{tabular}{|c|c|c|c|c|c|c|c|}
\hline \multirow[b]{3}{*}{ Name } & \multicolumn{5}{|c|}{ COVRP } & \multicolumn{2}{|c|}{ CVRP } \\
\hline & \multicolumn{2}{|c|}{ Root node } & \multicolumn{3}{|c|}{ Branch \& cut } & \multicolumn{2}{|c|}{ Branch \& cut } \\
\hline & LB & Time & LB & Tree size & Time & LB & Time \\
\hline B-n31-k5 & 360.287 & 0.3 & $362.725^{*}$ & 6 & 0.4 & $676.088^{*}$ & 0.9 \\
\hline B-n34-k5 & 452.184 & 6 & $458.764^{*}$ & 131 & 13 & $789.841^{*}$ & 7 \\
\hline B-n35-k5 & 557.326 & 0.1 & $557.326^{*}$ & 1 & 0.1 & $956.294^{*}$ & 0.04 \\
\hline B-n38-k6 & 445.583 & 0.1 & $445.628^{*}$ & 2 & 0.1 & $807.879^{*}$ & 2 \\
\hline B-n39-k5 & 317.76 & 0.6 & $322.539 *$ & 25 & 1 & $553.156^{*}$ & 0.2 \\
\hline B-n41-k6 & 480.432 & 0.4 & $483.068^{*}$ & 20 & 2 & $833.664^{*}$ & 5 \\
\hline B-n43-k6 & 428.167 & 0.4 & $428.167^{*}$ & 1 & 0.4 & $746.694^{*}$ & 7 \\
\hline B-n44-k7 & 499.447 & 2 & $501.308^{*}$ & 26 & 4 & $914.965^{*}$ & 0.4 \\
\hline B-n $45-\mathrm{k} 5$ & 484.19 & 5 & $488.065^{*}$ & 27 & 7 & $753.961^{*}$ & 4 \\
\hline B-n45-k6 & 390.288 & 1 & $403.812^{*}$ & 7645 & 1536 & $680.438^{*}$ & 21 \\
\hline B-n50-k7 & 437.151 & 0.2 & $437.151^{*}$ & 1 & 0.2 & $744.228^{*}$ & 0.6 \\
\hline B-n50-k8 & 703.571 & 4 & 720.427 & 12685 & 3600 & 1313.548 & 3600 \\
\hline B-n51-k7 & 620.415 & 1 & $625.14^{*}$ & 75 & 8 & $1035.04^{*}$ & 8 \\
\hline B-n52-k7 & 440.186 & 0.3 & $441.193^{*}$ & 12 & 0.8 & $749.97^{*}$ & 2 \\
\hline B-n56-k7 & 415.385 & 2 & $420.485^{*}$ & 208 & 17 & $712.916^{*}$ & 7 \\
\hline B-n57-k7 & 639.227 & 4 & $646.364^{*}$ & 979 & 228 & $1157.731^{*}$ & 152 \\
\hline B-n57-k9 & 854.691 & 4 & 868.025 & 12972 & 3600 & $1602.289^{*}$ & 585 \\
\hline B-n63-k10 & 826.616 & 3 & $837.072^{*}$ & 124 & 25 & $1499.096^{*}$ & 276 \\
\hline B-n64-k9 & 508.17 & 4 & $520.466^{*}$ & 1786 & 499 & $868.194^{*}$ & 14 \\
\hline B-n66-k9 & 747.287 & 4 & $755.274^{*}$ & 700 & 248 & 1320.771 & 3600 \\
\hline B-n67-k10 & 604.225 & 4 & 616.077 & 12939 & 3600 & $1039.268^{*}$ & 375 \\
\hline B-n68-k9 & 694.163 & 6 & $701.717^{*}$ & 1335 & 512 & 1273.673 & 3600 \\
\hline B-n78-k10 & 704.604 & 8 & 715.399 & 5778 & 3600 & 1225.716 & 3600 \\
\hline
\end{tabular}

Table 2: Results for the B instances.

\begin{tabular}{|c|c|c|c|c|c|c|c|}
\hline \multirow[b]{3}{*}{ Name } & \multicolumn{5}{|c|}{ COVRP } & \multirow{2}{*}{\multicolumn{2}{|c|}{$\frac{\text { CVRP }}{\text { Branch \& cut }}$}} \\
\hline & \multicolumn{2}{|c|}{ Root node } & \multicolumn{3}{|c|}{ Branch \& cut } & & \\
\hline & LB & Time & LB & Tree size & Time & LB & Time \\
\hline E-n22-k4 & 252.614 & 0.08 & $252.614^{*}$ & 1 & 0.08 & $375.28^{*}$ & 0.2 \\
\hline E-n23-k3 & 428.66 & 0.1 & $442.984^{*}$ & 22 & 0.3 & $568.563^{*}$ & 0.05 \\
\hline E-n30-k3 & 385.971 & 0.4 & $393.512^{*}$ & 56 & 1 & $535.797^{*}$ & 2 \\
\hline E-n33-k4 & 506.346 & 0.3 & $511.263^{*}$ & 8 & 0.6 & $837.672^{*}$ & 2 \\
\hline E-n51-k5 & 411.481 & 1 & $416.063^{*}$ & 102 & 16 & $524.611^{*}$ & 11 \\
\hline E-n76-k7 & 522.271 & 10 & $530.022^{*}$ & 2660 & 1103 & 682.563 & 3600 \\
\hline E-n76-k8 & 529.371 & 12 & $537.239 *$ & 1282 & 636 & 733.686 & 3600 \\
\hline E-n76-k10 & 547.826 & 21 & 559.233 & 4118 & 3600 & 818.655 & 3600 \\
\hline E-n76-k14 & 602.007 & 24 & 614.442 & 3178 & 3600 & 989.257 & 3600 \\
\hline E-n101-k8 & 633.851 & 6 & $639.744^{*}$ & 3378 & 2379 & 820.552 & 3600 \\
\hline E-n101-k14 & 692.149 & 33 & 699.985 & 1606 & 3600 & 1049.534 & 3600 \\
\hline F-n45-k4 & 463.896 & 0.1 & $463.896^{*}$ & 1 & 0.1 & $723.541^{*}$ & 1 \\
\hline F-n72-k4 & 175.924 & 9 & $176.999 *$ & 26 & 11 & $241.974^{*}$ & 4 \\
\hline F-n135-k7 & 753.431 & 27 & 762.894 & 1918 & 3600 & $1162.957^{*}$ & 1587 \\
\hline M-n101-k10 & 528.237 & 6 & $534.239^{*}$ & 297 & 89 & $819.558^{*}$ & 5 \\
\hline M-n121-k7 & 647.59 & 98 & 657.056 & 1046 & 3600 & 1034.782 & 3600 \\
\hline M-n151-k12 & 725.089 & 70 & 730.204 & 1354 & 3600 & 992.834 & 3600 \\
\hline
\end{tabular}

Table 3: Results for the E, F and M instances. 


\begin{tabular}{|c|c|c|c|c|c|c|c|}
\hline \multirow[b]{3}{*}{ Name } & \multicolumn{5}{|c|}{ COVRP } & \multirow{2}{*}{\multicolumn{2}{|c|}{$\frac{\text { CVRP }}{\text { Branch \& cut }}$}} \\
\hline & \multicolumn{2}{|c|}{ Root node } & \multicolumn{3}{|c|}{ Branch \& cut } & & \\
\hline & LB & Time & $\mathrm{LB}$ & Tree size & Time & LB & Time \\
\hline P-n16-k8 & 235.06 & 0.09 & $235.06^{*}$ & 1 & 0.09 & $451.335^{*}$ & 0.4 \\
\hline P-n19-k2 & 162.586 & 0.09 & $168.57^{*}$ & 12 & 0.2 & $212.657^{*}$ & 0.1 \\
\hline P-n20-k2 & 167.814 & 0.05 & $170.278^{*}$ & 12 & 0.2 & $217.416^{*}$ & 0.1 \\
\hline P-n21-k2 & 163.877 & 0.02 & $163.877^{*}$ & 1 & 0.02 & $212.712^{*}$ & 0.03 \\
\hline P-n22-k2 & 167.191 & 0.03 & $167.191^{*}$ & 1 & 0.03 & $217.852^{*}$ & 0.3 \\
\hline P-n22-k8 & 335.27 & 2 & $345.867^{*}$ & 22 & 3 & $600.826^{*}$ & 2 \\
\hline P-n23-k8 & 282.472 & 0.9 & $302.87^{*}$ & 428 & 36 & $531.174^{*}$ & 6 \\
\hline P-n40-k5 & 347.348 & 0.4 & $349.552^{*}$ & 19 & 2 & $461.726^{*}$ & 2 \\
\hline P-n45-k5 & 388.999 & 2 & $391.809^{*}$ & 36 & 4 & $512.791^{*}$ & 7 \\
\hline P-n50-k7 & 391.535 & 1 & $397.376^{*}$ & 222 & 24 & $559.863^{*}$ & 136 \\
\hline P-n50-k8 & 405.852 & 2 & 422.891 & 10014 & 3600 & 628.081 & 3600 \\
\hline P-n50-k10 & 426.604 & 2 & $440.438^{*}$ & 4704 & 1072 & 696.35 & 3600 \\
\hline P-n51-k10 & 455.056 & 3 & 472.858 & 9400 & 3600 & 741.499* & 2225 \\
\hline P-n55-k7 & 408.572 & 0.7 & $411.581^{*}$ & 60 & 7 & $570.27^{*}$ & 1599 \\
\hline P-n55-k8 & 410.584 & 2 & $412.554^{*}$ & 13 & 4 & $578.61^{*}$ & 207 \\
\hline P-n55-k10 & 433.56 & 2 & $444.308^{*}$ & 603 & 128 & 687.141 & 3600 \\
\hline P-n55-k15 & 522.954 & 8 & 544.341 & 5358 & 3600 & 932.103 & 3600 \\
\hline P-n60-k10 & 469.757 & 6 & $482.085^{*}$ & 2712 & 914 & 741.242 & 3600 \\
\hline P-n60-k15 & 542.407 & 11 & 560.345 & 5902 & 3600 & 952.636 & 3600 \\
\hline P-n65-k10 & 512.073 & 7 & $522.501^{*}$ & 2434 & 1059 & 789.344 & 3600 \\
\hline P-n70-k10 & 536.04 & 9 & 548.341 & 4670 & 3600 & 814.949 & 3600 \\
\hline P-n76-k4 & 516.857 & 4 & $522.945^{*}$ & 804 & 319 & $598.196^{*}$ & 37 \\
\hline P-n76-k5 & 516.57 & 10 & $525.635^{*}$ & 2721 & 1367 & $633.317^{*}$ & 3124 \\
\hline P-n101-k4 & 619.495 & 19 & $621.749^{*}$ & 56 & 45 & $691.287^{*}$ & 47 \\
\hline
\end{tabular}

Table 4: Results for the P instances.

\begin{tabular}{lccccc} 
Name & Our LB & Brandão & Fu et al. & Li et al. & P \& R \\
\hline E-n51-k5 & $416.1^{*}$ & $416.1^{*}$ & $416.1^{*}$ & $416.1^{*}$ & $416.1^{*}$ \\
E-n76-k10 & 559.2 & 574.5 & 567.1 & 567.1 & 567.1 \\
E-n101-k8 & $639.7^{*}$ & 641.6 & 641.9 & $639.7^{*}$ & 641.8 \\
M-n101-k10 & $534.2^{*}$ & 535.1 & 534.7 & $534.2^{*}$ & $534.2^{*}$ \\
M-n121-k7 & 657.1 & 683.4 & 717.2 & 682.5 & 682.1 \\
M-n151-k12 & 730.2 & 740.8 & 738.9 & 733.1 & 733.1 \\
M-n200-k16 & 848.5 & 953.4 & - & 925.0 & 896.1 \\
M-n200-k17 & 847.6 & - & 879.0 & - & - \\
F-n72-k4 & $177.0^{*}$ & 177.4 & $177.0^{*}$ & $177.0^{*}$ & $177.0^{*}$ \\
F-n135-k7 & 762.9 & 781.2 & 777.1 & 769.7 & 770.2 \\
\hline
\end{tabular}

Table 5: Lower bounds compared with upper bounds from the literature. 
In general, it appears that the open version is usually a little easier to solve than the closed version. A remarkable instance is $\mathrm{E}-\mathrm{n} 76-\mathrm{k} 8$, where the open version can be solved in about 10 minutes, whereas the closed version is known to be difficult. However, there are some exceptions, such as $\mathrm{F}-\mathrm{n} 135-\mathrm{k} 7$.

Finally, in table 5, we use our lower bounds to assess the quality of the existing heuristic methods for the COVRP. For each of 10 COVRP instances, the table reports the lower bound we obtained within 1 hour, followed by the upper bounds obtained by the four best heuristics, those of Brandão (2004), Fu, Eglese \& Li (2005), Li et al. (2006) and Pisinger \& Ropke (2006). It will be seen that the heuristics do rather well and actually produce optimal solutions in a few cases. (Note: some of the upper bounds in the Fu et al. column are different from those in $\mathrm{Fu}$, Eglese and $\mathrm{Li}$ (2005). This was due to a bug in the code, which has been corrected. A corrigendum has been submitted to the journal.)

Optimal solutions for all COVRP instances solved to optimality are available on the web site www.hha.dk/ lys/.

\section{Conclusion}

Although the COVRP appears to be a trivial variant of the standard CVRP, we have seen that it is intermediate in generality between the CVRP and the ACVRP. As a result, some subtle modifications are needed to adapt a branch-and-cut code for the CVRP to the COVRP. This includes modifications to the formulation, additional classes of inequalities, and adjustments to the separation algorithms.

Our results show that small- to medium-scale instances of the COVRP are just as amenable to exact solution by branch-and-cut as their CVRP counterparts. In fact, if anything, the open versions often appear to be slightly easier.

Future research could include the incorporation of column generation, leading to a full branch-cut-and-price algorithm along the lines of the one presented in Fukasawa et al. (2006). This would no doubt lead to the solution of even more instances, especially those with small vehicle capacities and a large number of vehicles. 


\section{References}

P. Augerat (1995) Approche Polyèdrale du Problème de Tournées de Véhicules. PhD thesis, Institut National Polytechnique de Grenoble.

L.D. Bodin, B.L. Golden, A.A. Assad and M.O. Ball (1983) Routing and scheduling of vehicles and crews: The state of the art. Computers $\&$ Operations Research, 10: 63-211. J. Brandão (2004) A tabu search algorithm for the open vehicle routing problem. Eur. J. Opl Res., 157: 552-564.

N. Christofides and S. Eilon (1969) An algorithm for the vehicle-dispatching problem. Operations Research Quarterly, 20: 309-318.

J. Edmonds (1965) Maximum matching and a polyhedron with 0-1 vertices. J. Res. Nat. Bur. Standards, 69B: 125-130.

M. Fischetti, P. Toth and D. Vigo (1994) A branch-and-bound algorithm for the capacitated vehicle routing problem on directed graphs. Oper. Res., 42: 846-859.

L. Ford and D. Fulkerson (1962) Flows in Networks. Princeton University Press, New Jersey.

Z. Fu, R. Eglese and L.Y.O. Li (2005) A new tabu search heuristic for the open vehicle routing problem. J. of the Opl Res. Soc., 56: 267-274.

R. Fukasawa, H. Longo, J. Lysgaard, M. Poggi de Aragão, M. Reis, E. Uchoa, and R.F. Werneck (2006) Robust branch-and-cut-and-price for the capacitated vehicle routing problem. Math. Program., 106: 491-511.

A.V. Goldberg and R.E. Tarjan (1988) A new approach to the maximum flow problem. Journal of the ACM, 35: 921-940.

G. Laporte, Y. Nobert and M. Desrochers (1985) Optimal routing under capacity and distance restrictions. Oper. Res., 33: 1050-1073.

A.N. Letchford, R.W. Eglese and J. Lysgaard (2002) Multistars, partial multistars and the capacitated vehicle routing problem. Math. Program., 94: 21-40.

F. Li, B. Golden and E. Wasil (2006) The open vehicle routing problem: algorithms, largescale test problems, and computational results. Comp. $E 3$ Oper. Res., to appear.

J. Lysgaard (2003) CVRPSEP: A package of separation routines for the capacitated ve- 
hicle routing problem. Working Paper 03-04, Department of Management Science and Logistics, Aarhus School of Business. Available at www.asb.dk/ lys/.

J. Lysgaard, A.N. Letchford and R.W. Eglese (2004) A new branch-and-cut algorithm for the capacitated vehicle routing problem. Math. Program., 100: 423-445.

D. Naddef and G. Rinaldi (2002) Branch-and-cut algorithms for the capacitated VRP. In Toth and Vigo, op. cit., chapter 3.

Y. Nobert and J.-C. Picard (1996) An optimal algorithm for the mixed chinese postman problem. Networks, 27: 95-108.

M. W. Padberg and M. R. Rao (1982) Odd minimum cut-sets and b-matchings. Math. Oper. Res., 7: 67-80.

D. Pisinger and S. Ropke (2006) A general heuristic for vehicle routing problems. Comp. Es Oper. Res., to appear.

G. Reinelt (1991) TSPLIB - A traveling salesman problem library. ORSA J. on Computing, 3: 376-384.

D. Sariklis and S. Powell (2000) A heuristic method for the open vehicle routing problem. J. of the Opl Res. Soc., 51: 564-573.

L. Schrage (1981) Formulation and structure of more complex/realistic routing and scheduling problems. Networks, 11: 229-232.

C.D. Tarantilis, G. Ioannou, C.T. Kiranoudis and G.P. Prastacos (2005) Solving the open vehicle routeing problem via a single parameter metaheuristic algorithm. J. of the Opl Res. Soc., 56: 588-596.

P. Toth and D. Vigo (eds.) (2002) The Vehicle Routing Problem. SIAM Monographs on Discrete Mathematics and Applications. 


\section{Appendix}

To show validity of the mixed comb inequalities, it is helpful to prove the following lemma.

Lemma 1 For any set $S$ such that $0 \in S$, the following three inequalities are valid.

$$
\begin{aligned}
& x(E(S \backslash\{0\}))+y^{+}(S \backslash\{0\})+y^{-}(S \backslash\{0\}) \leq|S|+K-k(V \backslash S)-1, \\
& x(E(S \backslash\{0\}))+y^{+}(S \backslash\{0\}) \leq|S|-1, \\
& x(E(S \backslash\{0\}))+y^{-}(S \backslash\{0\}) \leq|S|-1 .
\end{aligned}
$$

Proof: Due to the degree equations, the inequality (16) is equivalent to the capacity inequality on $V \backslash S$ and the inequalities (17) and (18) are equivalent to the balancing inequalities on $V \backslash S$ and $S \backslash\{0\}$, respectively.

Proof of Theorem 1: We follow the standard Chvátal-Gomory integer rounding argument. If we sum together the following inequalities:

the degree equations for all $i \in H$,

the inequality (6) on $H \cap T_{i}$ for $1 \leq i \leq t$,

the inequality (6) on $T_{i}$ and $T_{i} \backslash H$ for $i \in \mathcal{N}$,

the inequalities (16) for $T_{i}$ and $T_{i} \backslash H$, for $i \in \mathcal{D}$,

the inequalities (17) for $T_{i}$ and $T_{i} \backslash H$, for $i \in \mathcal{S}$, and

the inequalities (18) for $T_{i}$ and $T_{i} \backslash H$, for $i \in \mathcal{R}$,

we obtain (after some re-arranging):

$$
\begin{gathered}
2 x(E(H))+2 \sum_{i \in \mathcal{N}} x\left(E\left(T_{i}\right)\right)+2 \sum_{i \in \mathcal{D} \cup \mathcal{S} \cup \mathcal{R}} x\left(E\left(T_{i} \backslash\{0\}\right)\right)+2 \sum_{i \in \mathcal{D} \cup \mathcal{S}} y^{+}\left(T_{i} \backslash\{0\}\right) \\
+2 \sum_{i \in \mathcal{D} \cup \mathcal{R}} y^{-}\left(T_{i} \backslash\{0\}\right)+x\left(\delta(H) \backslash \bigcup_{i=1}^{t} E\left(T_{i}\right)\right)+y^{+}\left(H \backslash \bigcup_{i=1}^{t} T_{i}\right)+y^{-}\left(H \backslash \bigcup_{i=1}^{t} T_{i}\right) \\
\leq 2|H|+2 \sum_{i=1}^{t}\left|T_{i}\right|+2|\mathcal{D}|(K-1)-\sum_{i=1}^{t} \gamma\left(T_{i}\right) .
\end{gathered}
$$

Dividing this inequality by two and rounding down yields the result. 


\section{Working Papers from Logistics/SCM Research Group}

L-2006-06 Adam N. Letchford, Jens Lysgaard \& Richard W. Eglese: A Branch-andCut Algorithm for the Capacitated Open Vehicle Routing Problem.

L-2006-05 Ole Mortensen \& Olga W. Lemoine: Business integration between manufacturing and transport-logistics firms.

L-2006-04 Christian H. Christiansen \& Jens Lysgaard: A column generation approach to the capacitated vehicle routing problem with stochastic demands.

L-2006-03 Christian Larsen: Computation of order and volume fill rates for a base stock inventory control system with heterogeneous demand to investigate which customer class gets the best service.

L-2006-02 Søren Glud Johansen \& Anders Thorstenson: Note: Optimal base-stock policy for the inventory system with periodic review, backorders and sequential lead times.

L-2006-01 Christian Larsen \& Anders Thorstenson: A comparison between the order and the volume fill rates for a base-stock inventory control system under a compound renewal demand process.

L-2005-02 Michael M. Sørensen: Polyhedral computations for the simple graph partitioning problem.

L-2005-01 Ole Mortensen: Transportkoncepter og IT-støtte: et undersøgelsesoplæg og nogle foreløbige resultater.

L-2004-05 Lars Relund Nielsen, Daniele Pretolani \& Kim Allan Andersen: $K$ shortest paths in stochastic time-dependent networks.

L-2004-04 Lars Relund Nielsen, Daniele Pretolani \& Kim Allan Andersen: Finding the $K$ shortest hyperpaths using reoptimization.

L-2004-03 Søren Glud Johansen \& Anders Thorstenson: The $(r, q)$ policy for the lostsales inventory system when more than one order may be outstanding.

L-2004-02 Erland Hejn Nielsen: Streams of events and performance of queuing systems: The basic anatomy of arrival/departure processes, when focus is set on autocorrelation.

L-2004-01 Jens Lysgaard: Reachability cuts for the vehicle routing problem with time windows. 
ISBN 87-7882-123-1

Department of Business Studies

Aarhus School of Business

Fuglesangs Allé 4

DK-8210 Aarhus V - Denmark

Tel. +4589486688

Fax +4586150188

www.asb.dk 